\title{
Augmented Reality Controlled Smart Wheelchair Using Dynamic Signifiers for Affordance Representation
}

\author{
Rodrigo Chacón-Quesada and Yiannis Demiris ${ }^{1}$
}

\begin{abstract}
The design of augmented reality interfaces for people with mobility impairments is a novel area with great potential, as well as multiple outstanding research challenges. In this paper we present an augmented reality user interface for controlling a smart wheelchair with a head-mounted display to provide assistance for mobility restricted people. Our motivation is to reduce the cognitive requirements needed to control a smart wheelchair. A key element of our platform is the ability to control the smart wheelchair using the concepts of affordances and signifiers. In addition to the technical details of our platform, we present a baseline study by evaluating our platform through user-trials of able-bodied individuals and two different affordances: 1) Door - Go Through and 2) People - Approach. To present these affordances to the user, we evaluated fixed symbol based signifiers versus our novel dynamic signifiers in terms of ease to understand the suggested actions and its relation with the objects. Our results show a clear preference for dynamic signifiers. In addition, we show that the task load reported by participants is lower when controlling the smart wheelchair with our augmented reality user interface compared to using the joystick, which is consistent with their qualitative answers.
\end{abstract}

\section{INTRODUCTION}

Controlling a powered wheelchair can be a cognitively challenging task for some users [1]. To enable easier control of powered wheelchairs, a variety of control interfaces have been proposed; the most traditional method used are joysticks, but others include fingertip control, head tilt, electromyography and/or electroencephalogram signals [2], [3], [4], [5]. AR User Interfaces (UIs) have the potential of introducing more natural interactions between the user and the robot that are easier to understand and learn. Even though mobile Augmented Reality (AR) interfaces for wheelchairs have been previously proposed [6], [7], [8], to the best of our knowledge these interfaces have never been used before as a way to enable control, but instead mainly as a way of providing visual aids.

In this paper, we propose to use a head-mounted display (HMD) AR UI as a new method to controlling a smart wheelchair. For this, we integrated a smart wheelchair with an AR UI developed for the Microsoft HoloLens. Our platform jointly locates the objects and people in the smart wheelchair's surroundings and informs the users through the

\footnotetext{
${ }^{1}$ The authors are with the Personal Robotics Laboratory, Dept. of EEE, Imperial College London, SW7 2AZ, UK. \{r. chacon-quesada17, y.demiris\}@imperial.ac.uk.

Rodrigo Chacon-Quesada is supported by a PhD studentship jointly funded by the University of Costa Rica and Ministry of Science Technology and Telecommunications of Costa Rica. Yiannis Demiris is supported by a Royal Academy of Engineering Chair in Emerging Technologies. Lab info and videos: www.imperial.ac.uk/PersonalRobotics
}

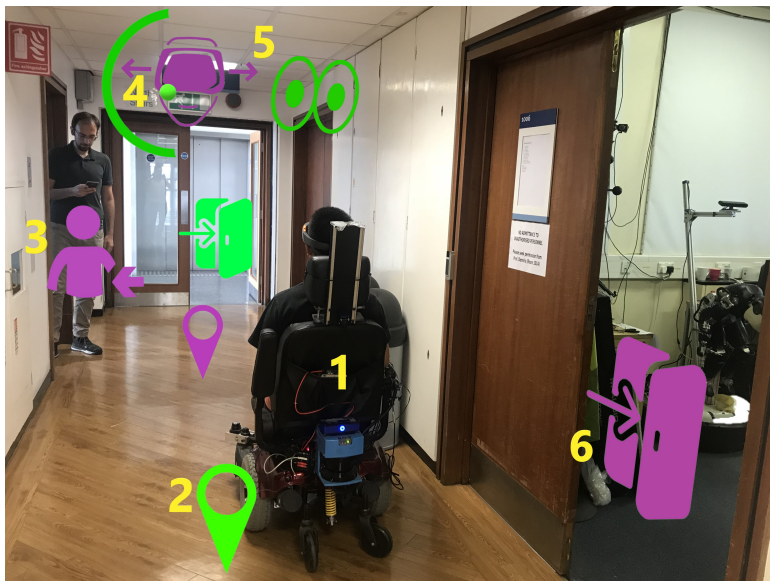

Fig. 1: Composite image of the visualisations rendered on the users view through the AR headset. (1) Smart Wheelchair. (2) We use the marker symbols to represent locations. (3) People - Approach affordances are placed when persons are detected. (4) The cursor and timing bar give feedback to the user during the aiming and selection stages respectively. (5) The different aiming methods available can be selected by the user using an on-board menu. (6) Door - Go Through affordances are shown when open doors are detected. Selected signifiers are shown in green and non-selected signifiers in purple.

use of signifiers about their affordances. Affordances are defined as the relationship between the properties of an object and the capabilities of an agent (whether human, animal or robot) that determine how the detected objects can be used to accomplish higher level tasks [9], [10]. For example, a door is signified as "passable" if its width characteristics are compatible with the size of the wheelchair. A composite image showing some of the visualisations rendered on the users view through the HMD is shown in Fig. 1

Our platform has the potential to serve a variety of mobility impairments, such as quadriplegia, amputations and cerebral palsy, given the multi-modality of input methods provided within our HMD UI, while at the same time aims at reducing the cognitive requirements needed to control the smart wheelchair by assisting the user in the perception and planning tasks naturally involve in this kind of activity.

\section{RELATED WORK}

\section{A. Virtual and Augmented Reality for Smart Wheelchairs}

To enable easier control of wheelchairs, a variety of control interfaces have been proposed. Leaman et al. [5] give 
a comprehensive survey about smart wheelchair research, and classify all available inputs methods in nine groups: Biometrics, Brain Computer Interface, Cloud, Computer Vision, Game Controller, Haptic Feedback, Multimodal, Touch and Voice. Virtual Reality (VR) technologies for smart wheelchair have been so far restricted to specific domains, such as simulation [11], [12] and training [13], [14], [15]. While mobile AR applications have also been researched, they have only been used as a way of giving additional information to the user, for example using fiducial markers [6] and beacon technologies [7] to provide the user with a route for indoor navigation. Zolotas et al. [8] have recently presented a novel AR-based interaction scenario, in which a wheelchair user can become more aware of the smart wheelchair's intentions in navigation tasks through a set of AR visualisations provided as feedback.

In this paper we enhance the VR/AR interaction to enable control of the smart wheelchair through an AR UI that informs the user about potential actions on objects and people in their surroundings using the concepts of affordances and signifiers [9], [10].

\section{B. User Interfaces for Interaction in Virtual and Augmented Environments}

Typically, two stages are needed to trigger an action while interacting with UIs in virtual and augmented environments: 1) the aiming stage, which is the process of positioning a cursor over a virtual object, and 2) the selection stage, which is the process of selecting the aimed virtual object.

For the aiming stage, external hand-held controllers have been proposed. The position and orientation of these controllers are tracked so that the user can aim at virtual objects. However, since the user needs to hold the controllers with their hands, the hands are unavailable to perform other actions. Alternatively, a widely used technique for the aiming stage is head-gaze. This technique measures the head direction of the user through a sensor in the VR / AR headset and displays a cursor in the centre of the user's view. The user then requires to move their head to control this cursor. Finally, eye-gaze is a promising handsfree interaction technique for the aiming stage and an active field of research [16], [17], [18], [19]. These techniques track the user's eyes with camera sensors and in combination with the head direction infers where the eyes are looking at in the environment. Eye-gaze has several advantages over headgaze in virtual environments in terms of speed, task load, required head movements and user preference [19]. However, when eye-gaze based techniques are used, it is more difficult for the users to remember the location of the virtual object they interact with, therefore making it less convenient for tasks that require spatial memory [17]. The eye-gaze aiming techniques are also affected by the "Midas touch" problem, defined as the difficulty to differentiate between gaze for perception or for user action [20]. In addition, it might lead to some technical issues, such as calibration quality and robustness, resulting in a limited application in non-research products [18].
The selection stage can be done using traditional techniques such as controller- or button-based selection methods, while more recent techniques are based on hand-gestures, voice commands, smooth pursuit [18], and dwell-time. In the latter, users need to remain aiming at the virtual object during a predefined amount of time to trigger the action. In this paper we propose a platform where multiple interaction techniques are provided to the user. It is important for our platform to be suitable for a wide variety of scenarios where a single interaction technique may not be enough. For example, voice commands may not be robust enough in noisy environments and not necessarily work well for people with speech impairments, hand-gestures could perform better in this case. Hand-gestures, however, are not available for people that due to their disability can not move their hands, and dwell-time could be applied instead.

\section{Affordances, Signifiers and Environmental Graphic De- sign}

Affordances and signifiers are closely related concepts. Affordances represent the interaction possibilities in the world; they are relationships between the agent and the object, and not stand-alone properties of the object [9]. Signifiers are defined as signals (signs, symbols, marks, sounds or any perceivable indicator) that signify meaningful information to explain what actions are possible, how they should be done and where an interaction should take place [10].

Symbols, which are graphic devices that communicate information without words, can be used as signifiers. Their meaning has the potential to transcend language barriers and sometimes communicate more clearly and concisely than words [21]. However, to avoid symbols failing to function, they must be perceivable and easy to understand [10], [21].

To achieve this perceivability and ease of understanding, there are some relevant guidelines from the field of environmental graphic design. Popular designs of symbol vocabularies include the AIGA/DOT [22], which is the most commonly use symbol vocabulary in the United States, and the Accessibility Symbols made by the Society for Experiential Graphic Design [23].

In this paper, we present a platform that gives the user the ability to control a smart wheelchair through an AR UI based around automatically inferred affordances. We signify these affordances through the use of symbol based signifiers following recommendations coming from the field of environmental graphic design. We assert that the interpretability of signifiers can be enhanced through movement; in this paper we have designed a set of novel dynamic signifiers, which are dynamic symbols that are experimentally shown to better explain to the user what actions are possible.

\section{SYSTEM DESCRIPTION}

To present the users of our system with a set of affordances, we developed an AR platform for controlling a smart wheelchair using signifiers to represent affordances. We used the Microsoft HoloLens to display these signifiers. An illustration of our platform's hardware and software 


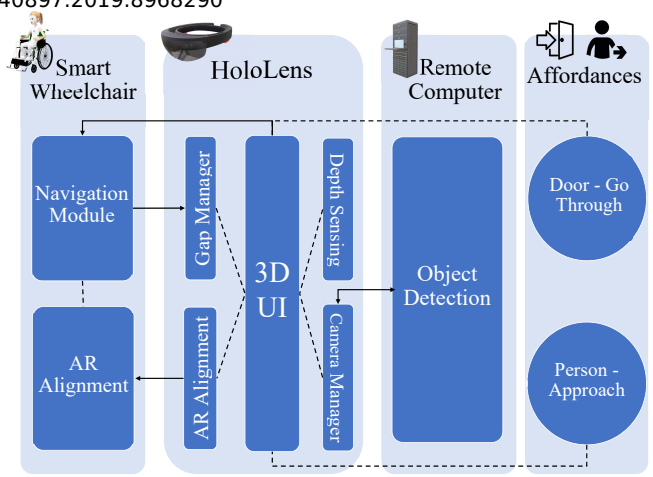

Fig. 2: System architecture. Rounded rectangles represent software nodes and circles are signifiers displayed in the HMD. Bold lines represent connections handle by the ROS Manager module and dashed lines represent connections within the same hardware module.

components is shown in Fig. 2, all of which communicate between each other using ROS.

\section{A. Smart Wheelchair}

Our smart wheelchair platform is based on a electric powered wheelchair, is interfaced with a laptop via an Arduino Uno to intercept and inject joystick inputs. Extra sensors have been added to the wheelchair, which include a Phidgets spatial 3/3/3 IMU unit, two Hokuyo URG-04LX-UG1 scanning laser range-finders situated at the front, and one SICK LMS200 rangefinder at the back. These sensors comprise the input to the Navigation Module which is composed by a collection of ROS packages. This Navigation Module uses a "gap-based" algorithm for reactive obstacle avoidance, which finds admissible gaps [24]. Furthermore, this module has the capability to receive location points towards which the smart wheelchair will autonomously navigate. The $3 D U I$ uses this capability to set the positions where the smart wheelchair is required to go when the user selects an applicable signifier. In addition, the built-in shared control given by this module manages the obstacle avoidance and local path planning tasks. The shared control method used by our platform is described in detail by Zolotas et al. in [8].

\section{B. Head-mounted Display}

The software modules running on the HMD were developed using Unity3D for the Microsoft HoloLens. The $3 D$ $U I$ module manages the interaction techniques available. For the aiming stage we are currently using head-gaze. For the selection stage, the user can choose between three different methods: hand-gestures, voice commands and dwell-time. All three selection methods are always available to the user without the need of explicitly selecting the preferred one. Dwell-time enables an extra hands-free way of interacting with the $3 D U I$ when combined with head-gaze, and offers the potential to serve people with mobility limitations that do not allow them to move more than their head. We evaluated this combination of interaction methods later in our experiments.

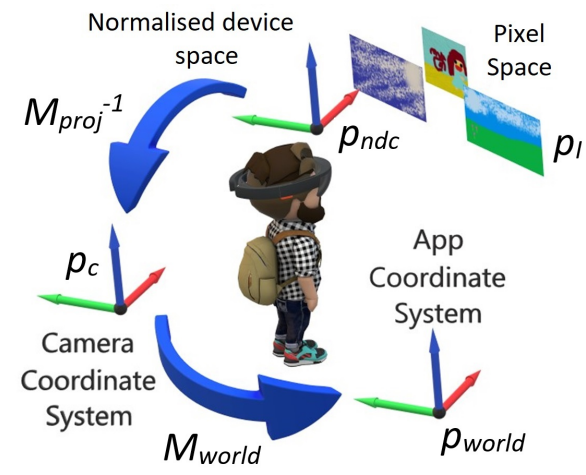

Fig. 3: Pixel Space to Application coordinate system transformations. We use the semantic information obtained by using images in conjunction with a set of transformations for the 3D placement of holograms.

The $3 D U I$ also provides feedback to the user in both the aiming and selection stages. A red dot that shows the user's gaze position is used as a cursor that changes its colour from red to green when a signifier is targeted. All signifiers change their colour when selected and a timing bar is shown for the dwell-time selection method. The detected affordances are shown to the user in the location of the associated object using signifiers to signify the actions that can be performed. To place these signifiers, the $3 D U I$ uses the information coming from the Camera Manager, Depth Sensing and the Gap Manager modules which provide information about the location of the objects in the 3D space. The ROS Manager connects the HoloLens to rosbridge using an API based on the work presented by Codd-Downey et al. in [25].

The AR Alignment module handles the correspondence between the frames of reference of the HMD and the smart wheelchair. This module uses three virtual objects as spatial anchors placed in the environment at known positions in a pre-built map. Spatial anchors represent important points in the world that the HoloLens keeps track of over time so that they stay precisely in place [26]. These positions are periodically reported by the virtual objects inside the application and compared to their corresponding positions in the ROS map to obtain the transformation between the application coordinate system and the smart wheelchair's frame of reference as described by Zolotas et al. [8].

The Camera Manager takes pictures using the HMD's camera, sends these pictures to the Object Detection module (discussed in Sec. IIII-C) and performs pixel to application coordinate system transformations to place signifiers associated with the detected affordances in the 3D environment. The acquisition process starts when the user instructs the platform to take a picture of the scene using hand-gestures or voice commands. After the picture is processed by the Object Detection module, the Camera Manager receives the pixel position $p_{I}=\left(x_{I}, y_{I}\right)$ of all identified objects within the image $I$. This position is then transformed to normalised device coordinates (ndc) using a simplified viewport matrix and the following equation [27]: 



(a)

(b)

Fig. 4: An illustration of the incorporated affordances. (a) Door - Go Through. (b) People - Approach.

$$
\left(x_{I}, y_{I}, z_{n d c}, 1\right)=p_{n d c} \cdot\left[\begin{array}{cccc}
\frac{\text { width }}{2} & 0 & 0 & 0 \\
0 & \frac{\text {-height }}{2} & 0 & 0 \\
0 & 0 & 1 & 0 \\
\frac{\text { width }}{2} & \frac{\text { height }}{2} & 0 & 1
\end{array}\right]
$$

Where width and height corresponds to the image resolution width and height of the image $I$ respectively and $p_{n d c}=\left(x_{n d c}, y_{n d c}, z_{n d c}, w\right)$. In the HoloLens' camera the image plane is located one unit away from the origin, this is $z_{n d c}=1$, and a value of 1 is used for the $w$ component [28]. Solving the previous equation for $p_{n d c}$ yields:

$$
p_{n d c}=\left(\frac{2 \cdot x_{I}}{\text { width }}-1,-\frac{2 \cdot y_{I}}{h e i g h t}+1,1,1\right)
$$

With the coordinates of $p_{I}$ in $n d c$, we can now find the position of the pixel in the camera coordinate system by using the camera projection matrix as follows:

$$
p_{c}=p_{n d c} \cdot M_{\text {proj }}^{-1}
$$

where $M_{\text {proj }}{ }^{-1}$ is the inverse of the projection matrix and $p_{c}=\left(x_{c}, y_{c}, z_{c}, 1\right)$ is the position of $p_{n d c}$ in the camera coordinate system. Equations (1) to (3) require a fixed frame of reference at time of image acquisition and thus the user is needed to remain still. The position of the camera in the camera coordinate system $o_{c}=(0,0,0,1)$ and $p_{c}$ are then transformed into the application coordinate system using a camera to world transformation matrix $M_{\text {world }}$ to find $o_{w o r l d}$ and $p_{\text {world }}$.

Both $M_{\text {proj }}$ and $M_{\text {world }}$ are provided by the HoloLens for each frame. We illustrate this transformation process in Fig. 3 As the depth image is (by default) not available using the HoloLens, a ray tracing technique is used instead; a ray representing the path taken by the photons to produce the pixel is cast using $p_{\text {world }}$ and $o_{\text {world }}$ to find the position where it hits the spatial mapping mesh obtained by the Depth Sensing module, this position corresponds to the 3D position of $p_{I}$ [29]. By using the transform from the HMD's coordinate system to the smart wheelchair's coordinate system obtained by the AR Alignment module this 3D position can be set as a location point towards which the smart wheelchair will navigate to.

The Gap Manager receives the gaps obtained by means of the Navigation Module and filters them based on their width to decide if the gaps being analysed correspond to doors. If



(f)

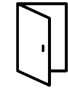

(b)

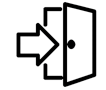

(c)

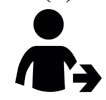

(g) (h)

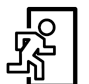

(d)

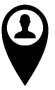

(i)

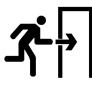

(e)

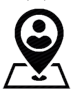

(j)
Fig. 5: Symbols used in our survey. Symbols (a) to (e) were used for the Door - Go Through affordance while symbols (a) and (f) to (j) were used for the People - Approach affordance.

the gap is classified as a door the Gap Manager sends the corresponding location to the $3 D U I$ to place the associated signifier in the $3 \mathrm{D}$ environment.

\section{Remote computer}

To achieve a stable display frame-rate of $60 \mathrm{fps}$ while dealing with long-running image recognition algorithms, the Object Detection module runs as a service in a remote computer that receives the pictures taken by the Camera Manager. The Object Detection module represents each detected object with the centroid of their corresponding boundingboxes and their labels. This information is then send to the Camera Manager. The current version of our platform uses Detectron to perform the object detection task in pictures [30]. The connection with this remote computer requires our platform to be cloud-connected. However, by adding an onboard computer with enough embedded processing power the system could be kept as stand-alone.

\section{Affordances}

A key element of our platform is the possibility to control a smart wheelchair through an AR UI that uses the concepts of affordances and signifiers discussed in Sec. II-C to suggest higher level actions to the user. This means that our platform needs to consider its own capabilities as well as the objects present in the scene before suggesting such actions. Following this idea, we integrated the affordances into our platform as shown in Fig. 4, specifically actions Go Through and Approach depends on the capability of the smart wheelchair to move. A signifier suggesting the actions will be instantiated through the HMD in the appropriate location. If the user selects the signifier, the position of the object will be sent to the wheelchair's navigation system and the smart wheelchair will autonomously navigate towards this position. Door and Person are object categories that our platform is able to detect using different sources of semantic information (laser scanner readings and images) and at the same time allowed us to illustrate two different type of actions (Go Through and Approach). By including more of these object categories together with actions that require moving from one place to another, more affordances can be incorporated. Some future affordances that could be implemented without adding modules to our platform are: Bicycle - Follow, Bus - Get On, Dining Table - Sit, among others. 
TABLE I: Demographics of the survey participants and the number of times each symbol was selected $(N=44)$.

\begin{tabular}{lcc}
\hline Factor & $n$ & $\%$ \\
\hline Gender & & \\
Female & 13 & 29.5 \\
Male & 31 & 70.5 \\
Age (years) & & \\
$<18$ & 5 & 11.4 \\
$18-24$ & 8 & 18.2 \\
$25-34$ & 18 & 40.9 \\
$35-44$ & 9 & 20.5 \\
$45-54$ & 3 & 6.8 \\
$55-64$ & 1 & 2.3 \\
Region & & \\
Africa & 1 & 2.3 \\
America & 31 & 70.5 \\
Asia & 1 & 2.3 \\
Europe & 11 & 25.0 \\
\hline
\end{tabular}

\begin{tabular}{ccc}
\hline Affordance & $n$ & $\%$ \\
\hline Door-Go Through & & \\
Fig. $5 \mathrm{5a}$ & 0 & 0.0 \\
Fig. & 2 & 4.5 \\
Fig. & 19 & 43.2 \\
Fig. & 5 & 11.4 \\
Fig. & 18 & 40.9 \\
Othe & 0 & 0.0 \\
Person-Approach & & \\
Fig. & 1 & 2.3 \\
Fig. & 8 & 18.2 \\
Fig. & 0 & 0.0 \\
Fig. & 11 & 25.0 \\
Fig. & 10 & 22.7 \\
Fig. & 9 & 20.5 \\
Other & 5 & 11.4 \\
\hline
\end{tabular}

TABLE II: Demographics of the experiment participants and their reported experience with other technologies $(N=16)$.

\begin{tabular}{lcc}
\hline Factor & $n$ & $\%$ \\
\hline Gender & & \\
Female & 5 & 31 \\
Male & 11 & 69 \\
Age (years) & & \\
18-24 & 2 & 12.5 \\
$25-34$ & 14 & 87.5 \\
Group & & \\
Exp. & 8 & 50 \\
Non-exp & 8 & 50 \\
\hline
\end{tabular}

\begin{tabular}{lcc}
\hline Baseline & $n$ & $\boldsymbol{\%}$ \\
\hline Exp. & & \\
VR & 8 & 100 \\
AR & 7 & 87.5 \\
Computer games & 8 & 100 \\
Non-exp. & & \\
VR & 4 & 50 \\
AR & 2 & 25 \\
Computer games & 8 & 100 \\
\hline
\end{tabular}

\section{EXPERIMENTAL PROCEDURE AND EVALUATION}

\section{A. Survey}

As cultural differences can affect symbol recognition and understanding [21], we decided to design a survey where we asked people from different cultural backgrounds to choose from a given set of symbols the one that they think better describes the affordances Door - Go Through and People - Approach. If none of the given symbols satisfied the participants, they had the option to add a description for a symbol they would prefer. The symbols used for the survey are shown in Fig. 5 and were chosen to be stylistically compatible with the Society for Experiential Graphic Design [23] and AIGA/DOT [22] symbols. The demographics of the participants and a summary of the results of the survey are shown in Table []

Based on the results obtained, the symbols shown in Fig. $5 \mathrm{c}$ and Fig. 5h were selected to represent the affordances. Furthermore, we noticed how symbols including doors and persons and in addition suggesting movement were among the favourites for participants. This result motivated us to enhanced the fixed symbols with interpretative information. As a result, we made a set of signifiers that periodically iterate images inside a loop giving a sense of movement (a behaviour similar to an animated GIF). We call these animated symbols dynamic signifiers. We compared the effectiveness of our novel dynamic signifiers later in our experiments.

\section{B. Experimental Setup}

We performed an evaluation of our platform with 16 ablebodied participants. In order to provide a comparison baseline we used joystick, although this control method would not be available to many disabled people where our platform would be their only option. We compared this control method against our proposed AR UI using head-gaze and dwelltime, the combination of these two interactions methods was selected for convenience, as they provide a hands-free interaction with the interface simulating a disability where the user is not able to move their hands. We made this comparison in terms of perceived ease of use, reported cognitive workload using NASA (raw) TLX scores, and time to complete the task (retrieved using the HMD). Participants were separated in two groups: experienced users and nonexperienced users. Non-experienced users were defined as having no prior experience with wheelchairs. Experienced users were defined as having previously participated in experiments from our laboratory requiring the use of smart wheelchair and/or smart wheelchair with visual feedback. No training sessions were given. Consent was obtained from the participants followed by a demographics and baseline questionnaire.

To start, the interpupillary distance was calibrated for each user using the built-in application from the HoloLens for this purpose. The first task of the experiment was intended to compare the use of fixed symbols against our proposed dynamic signifiers in terms of user preference. For this, participants were asked to describe the actions being suggested by the signifiers using fixed icons. Then, the same affordances were shown to the participants using dynamic signifiers.

For the following two tasks, participants needed to complete a trial route that required them to go through a door. The start position was set using a virtual marker represented with the symbol shown in Fig. 5g. For the second task, participants were asked to complete the trial route using the joystick. Participants were required to wear the HoloLens at all times. For the third task, participants were asked to complete the trial route using our AR UI with head-gaze as aiming method and dwell-time as selection method, simulating a disability of the hands. In addition, a virtual marker represented with the symbol shown in Fig. $5 \mathrm{~g}$ was placed in approximately half the trials. Participants first needed to select this marker to reach a position within the range of detection of the signifier associated with the door but without any need of using the joystick. Then, participants needed to select this last signifier and wait for the smart wheelchair to autonomously take them through the door.

Finally, a questionnaire asking participants about the driving experience and their visualisation preferences between fixed symbols of dynamic signifiers was filled; an average session took 30 minutes to complete.

Statistical analysis is performed using two group comparison test (i.e. T-Test). Descriptive statistics are represented according to normality tested using the KolmogorovSmirnov 

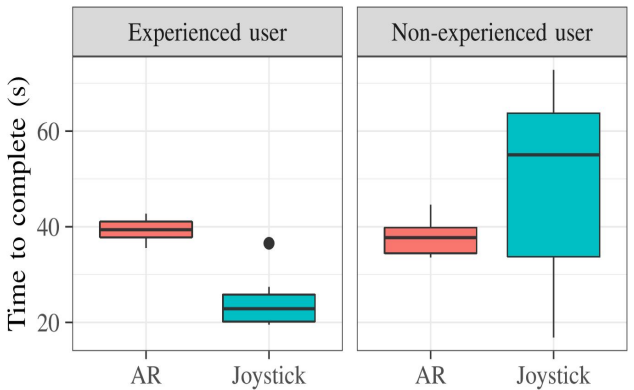

(a)

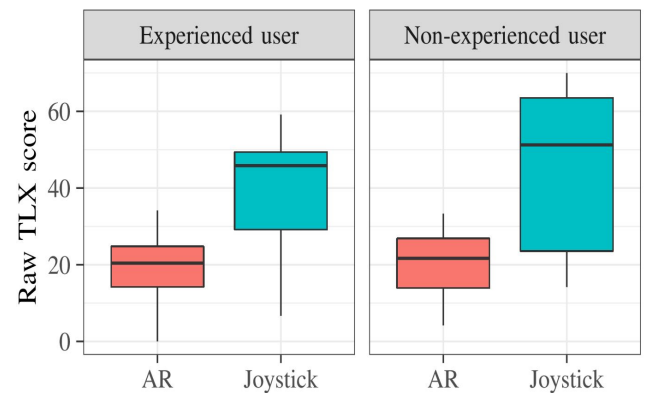

(b)

Fig. 6: Experimental Results. (a) Time to complete each task for each group and (b) Task load reported by participants.

TABLE III: Time to complete for non-exp. participants.

\begin{tabular}{lccc}
\hline ID & Joystick (s) & AR UI (s) & Improvement $(\boldsymbol{\%})$ \\
\hline PRL7 & 52.74 & 33.57 & 36 \\
PRL9 & 71.25 & 41.93 & 41 \\
PRL11 & 57.34 & 44.62 & 22 \\
PRL12 & 38.87 & 34.53 & 11 \\
PRL13 & 61.23 & 38.21 & 38 \\
PRL14 & 16.83 & 34.25 & $(104)$ \\
PRL16 & 18.37 & 39.12 & $(113)$ \\
PRL17 & 72.81 & 37.25 & 49 \\
\hline
\end{tabular}

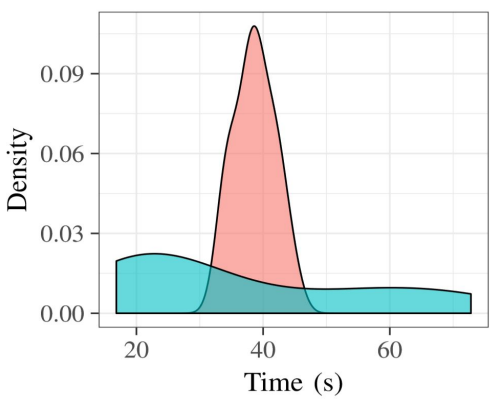

Input

$\square$ AR

Fig. 7: Time density distributions for each control method.

test. Normally distributed values are represented as means and standard deviations and non-normal distributed values represented as medians and inter-quartile ranges. The TLX questionnaire post experiment is analysed using a Wilcoxon signed-rank tests with Bonferroni-Holm correction.

\section{Experimental Results and Discussion}

The demographics of the participants and a summary of their reported experience with VR, AR and computer games are shown in Table [I] In Fig. 6a and Fig. 7 we summarise the time related results for both groups. Regarding the task load, in Fig. 6b we show a summary of the results obtained.

The results for the first task showed that $87.5 \%$ of the participants in each group preferred the use of dynamic signifiers. Only one participant from each group chose the fixed symbols because they found them less distracting. The rest of the participants found dynamic signifiers more clear, intuitive and meaningful. Due to the positive acceptability of our dynamic signifiers among the participants of our experiments, we will signify affordances incorporated in the future to our platform using dynamic signifiers. These will contain a representation of the object of interaction as well as arrows to reinforce directionality.

Importantly for the aims of this paper, the TLX score reported by the experienced users was lower for the task of controlling the smart wheelchair with our AR UI (19.5, $\mathrm{SD}=11.0)$ than when using the joystick (39.1, $\mathrm{SD}=$ 17.3). The same result occurred with the non-experienced users that reported a TLX score of $19.9(\mathrm{SD}=10.1)$ when using the AR UI and 44.79 ( $\mathrm{SD}=22.9$ ) when using the joystick. TLX analysis revealed that the difference in task load reported for each control method in both participant groups is significant at $\mathrm{p}<.01$. This is consistent with the qualitative answers obtained, $100 \%$ of the participants in the experienced users group chose the AR UI when asked about the method they think was easier to use for controlling the smart wheelchair. The result was similar for the non-experienced users group, seven out of eight participants chose the AR UI. Only one user chose the joystick and gave as reason his wide experience with video game controllers, this user also resulted to be the fastest between all participants when using the joystick. Reasons for preferring AR UI by the rest of participants from both groups included easiness of use, confidence, precision, amount of skills and commands needed and less effort required.

An additional interesting result was that experienced users were faster than non-experienced users when driving the smart wheelchair with the joystick, $24.3 \mathrm{~s}(\mathrm{SD}=5.7)$ and $48.7 \mathrm{~s}(\mathrm{SD}=21.9)$ respectively. However, this advantage was reduced when using our AR UI: the time to complete the task when controlling the smart wheelchair with the AR UI was similar between groups, experienced users finished the task in $39.4 \mathrm{~s}(\mathrm{SD}=2.4)$ and non-experienced users in $37.9 \mathrm{~s}$ (SD $=3.9$ ). A t-test showed that there was a significant difference between the experienced and non-experienced users $(\mathrm{p}<.02)$ when controlling the smart wheelchair using the joystick, while it did not show any significant differences between time values for both types of users when controlling the smart wheelchair using the AR UI. Experienced users were significantly slower $(\mathrm{p}<5.25 \mathrm{e}-5)$ with our AR UI than with the joystick. The main reason for this is that when using the AR UI these users had less control over how fast they could finish the task as the speed of the smart 
wheelchair is set by the local path planner (Sec. III-A) and not by the users through the joystick. For the same reason the variance in the time to complete the task for non-experienced users was reduced when using the AR UI. Although the difference in not statistically significant, our AR UI improved the execution times for six non-experienced participants as shown in Table III Our motivation is to reduce the cognitive requirements needed to control a smart wheelchair and the obtained results confirms that our platform allows easier control of the smart wheelchair for both experienced and new users at the expense of reducing the speed of the former ones.

\section{CONCLUSION}

In this paper we presented a novel HMD AR UI for controlling a smart wheelchair for people with limited mobility; we employed a HMD and an AR UI based on affordance perception and environmental graphic design theory to build our platform. We presented the concept of dynamic signifiers as a result of the survey we performed to choose the most appropriate symbols to represent the affordances we evaluated in this work, and showed that this results in a significant improvement in the ease of control of the wheelchair. While participants of our baseline study were all able-bodied and not representative of the target population, we have hopefully demonstrated sufficient evidence that the proposed platform can serve as a solution for this type of application.

We have also completed the implementation of an eyegaze method to replace the head-gaze input, to further reduce the motor input requirements of our system, and widen its applicability to people with different motor impairments. As future work, we will evaluate how people with different motor impairments benefit from all available interaction methods with the AR UI. In addition, as we have only evaluated examples of affordances involving moving to a particular location, in the future we will be incorporating more affordances to our platform by including more objects and their associated actions.

\section{REFERENCES}

[1] S. Massengale, D. Folden, P. McConnell, L. Stratton, and V. Whitehead, "Effect of visual perception, visual function, cognition, and personality on power wheelchair use in adults," Assistive Technology, vol. 17, no. 2, pp. 108-121, 2005.

[2] Y. Demiris, "Knowing when to assist: Developmental issues in lifelong assistive robotics," in 2009 Annual international conference of the IEEE engineering in medicine and biology society. IEEE, 2009, pp. 3357-3360.

[3] H. Soh and Y. Demiris, "Towards Early Mobility Independence : An Intelligent Paediatric Wheelchair with Case Studies," in Proceedings of the IROS 2012 Workshop on Progress, Challenges and Future Perspectives in Navigation and Manipulation Assistance for Robotic Wheelchairs, Algarve, Portugal., 2012.

[4] —_, "Learning assistance by demonstration: Smart mobility with shared control and paired haptic controllers," Journal of Human-Robot Interaction, vol. 4, no. 3, pp. 76-100, 2015.

[5] J. Leaman and H. M. La, "A comprehensive review of smart wheelchairs: Past, present, and future," IEEE Transactions on HumanMachine Systems, vol. 47, no. 4, pp. 486-499, Aug 2017.

[6] L. C. d. Oliveira, A. B. Soares, A. Cardoso, A. d. O. Andrade, and E. A. Lamounier Júnior, "Mobile augmented reality enhances indoor navigation for wheelchair users," Research on Biomedical Engineering, vol. 32, no. 2, pp. 111-122, 2016.
[7] L. C. d. Oliveira, A. O. Andrade, E. C. de Oliveira, A. Soares, A. Cardoso, and E. Lamounier, "Indoor navigation with mobile augmented reality and beacon technology for wheelchair users," in Biomedical \& Health Informatics (BHI), 2017 IEEE EMBS International Conference on. IEEE, 2017, pp. 37-40.

[8] M. Zolotas, J. Elsdon, and Y. Demiris, "Head-mounted augmented reality for explainable robotic wheelchair assistance," in 2018 IEEE/RSJ International Conference on Intelligent Robots and Systems (IROS), Oct 2018, pp. 1823-1829.

[9] J. J. Gibson, The senses considered as perceptual systems. Boston, MA, USA: Houghton Mifflin, 1966.

[10] D. Norman, The design of everyday things: Revised and expanded edition. Constellation, 2013.

[11] G. Di Gironimo, G. Matrone, A. Tarallo, M. Trotta, and A. Lanzotti, "A virtual reality approach for usability assessment: case study on a wheelchair-mounted robot manipulator," Engineering with Computers, vol. 29, no. 3, pp. 359-373, 2013.

[12] I. Ktena, W. Abbott, and A. A. Faisal, "A virtual reality platform for safe evaluation and training of natural gaze-based wheelchair driving," IEEE Neural Eng (NER), vol. 7, pp. 236-239, 2015.

[13] L. Devigne, M. Babel, F. Nouviale, V. K. Narayanan, F. Pasteau, and P. Gallien, "Design of an immersive simulator for assisted power wheelchair driving," in Rehabilitation Robotics (ICORR), 2017 International Conference on. IEEE, 2017, pp. 995-1000.

[14] A. Alshaer, H. Regenbrecht, and D. OHare, "Immersion factors affecting perception and behaviour in a virtual reality power wheelchair simulator," Applied ergonomics, vol. 58, pp. 1-12, 2017.

[15] N. W. John, S. R. Pop, T. W. Day, P. D. Ritsos, and C. J. Headleand, "The implementation and validation of a virtual environment for training powered wheelchair manoeuvres," IEEE transactions on visualization and computer graphics, vol. 24, pp. 1867-1878, 2018.

[16] T. Fischer, H. Jin Chang, and Y. Demiris, "Rt-gene: Real-time eye gaze estimation in natural environments," in Proceedings of the European Conference on Computer Vision (ECCV), 2018, pp. 334-352.

[17] V. Tanriverdi and R. J. Jacob, "Interacting with eye movements in virtual environments," in Proceedings of the SIGCHI conference on Human Factors in Computing Systems. ACM, 2000, pp. 265-272.

[18] S. Schenk, P. Tiefenbacher, G. Rigoll, and M. Dorr, "Spock: A smooth pursuit oculomotor control kit," in Proceedings of the 2016 CHI Conference Extended Abstracts on Human Factors in Computing Systems. ACM, 2016, pp. 2681-2687.

[19] J. Blattgerste, P. Renner, and T. Pfeiffer, "Advantages of eye-gaze over head-gaze-based selection in virtual and augmented reality under varying field of views," in Proceedings of the Workshop on Communication by Gaze Interaction. ACM, 2018, p. 1.

[20] R. J. Jacob, "Eye tracking in advanced interface design," Virtual environments and advanced interface design, pp. 258-288, 1995.

[21] C. Calori and D. Vanden-Eynden, Signage and wayfinding design: a complete guide to creating environmental graphic design systems. John Wiley \& Sons, 2015.

[22] American Institute of Graphic Arts, "Symbol signs," https://www.aiga. org/symbol-signs 2018, accessed: 2019-07.

[23] Society for Experiential Graphic Design, "Accessibility symbols," https://segd.org/accessibility-symbols 2014, accessed: 2019-07.

[24] M. Mujahed, D. Fischer, and B. Mertsching, "Admissible gap navigation: A new collision avoidance approach," Robotics and Autonomous Systems, vol. 103, pp. 93-110, 2018.

[25] R. Codd-Downey, P. M. Forooshani, A. Speers, H. Wang, and M. Jenkin, "From ros to unity: Leveraging robot and virtual environment middleware for immersive teleoperation," in Information and Automation (ICIA), 2014 IEEE International Conference on. IEEE, 2014, pp. 932-936.

[26] Microsoft, "Spatial anchors," https://docs.microsoft.com/en-us/ windows/mixed-reality/spatial-anchors 2019, accessed: 2019-07.

[27] F. D. Luna, Introduction to 3D game programming with DirectX 12. Mercury Learning \& Information, 2016.

[28] Microsoft, "Locatable camera," https://docs.microsoft.com/en-us/ windows/mixed-reality/locatable-camera 2019, accessed: 2019-07.

[29] - "Spatial mapping," https://docs.microsoft.com/en-us/windows/ mixed-reality/spatial-mapping 2018, accessed: 2019-07.

[30] R. Girshick, I. Radosavovic, G. Gkioxari, P. Dollár, and K. He, "Detectron," 2018. [Online]. Available: https://github. com/facebookresearch/detectron 